\title{
Identification and characterization of anion binding sites in RNA
}

\author{
JEFFREY S. KIEFT, ${ }^{1}$ ELAINE CHASE, ${ }^{2}$ DAVID A. COSTANTINO, ${ }^{1}$ and BARBARA L. GOLDEN ${ }^{2}$ \\ ${ }^{1}$ Howard Hughes Medical Institute and Department of Biochemistry and Molecular Genetics, University of Colorado School of Medicine, \\ Aurora, Colorado, 80045, USA \\ ${ }^{2}$ Department of Biochemistry, Purdue University, West Lafayette, Indiana 47907, USA
}

\begin{abstract}
Although RNA molecules are highly negatively charged, anions have been observed bound to RNA in crystal structures. It has been proposed that anion binding sites found within isolated RNAs represent regions of the molecule that could be involved in intermolecular interactions, indicating potential contact points for negatively charged amino acids from proteins or phosphate groups from an RNA. Several types of anion binding sites have been cataloged based on available structures. However, currently there is no method for unambiguously assigning anions to crystallographic electron density, and this has precluded more detailed analysis of RNA-anion interaction motifs and their significance. We therefore soaked selenate into two different types of RNA crystals and used the anomalous signal from these anions to identify binding sites in these RNA molecules unambiguously. Examination of these sites and comparison with other suspected anion binding sites reveals features of anion binding motifs, and shows that selenate may be a useful tool for studying RNA-anion interactions.
\end{abstract}

Keywords: RNA structure; anion binding; ion-RNA interactions; X-ray diffraction; anomalous difference; selenate

\section{INTRODUCTION}

In crystal structures of RNA molecules, a variety of solvent and buffer components are observed to bind at discrete sites. This includes water molecules that form hydration spines in both the major and minor grooves (Auffinger and Westhof 1998; Sundaralingam and Pan 2002) and cations that interact directly with functional groups from the nucleic acid and indirectly through water-mediated contacts (Pyle 2002). The positive charge of these ions neutralizes the overall negative charge of the phosphates and is critical for stabilizing the folds of biologically functional RNAs (Draper 2004; Woodson 2005). Less well-characterized are the interactions of negatively charged anions with nucleic acids. Localized

Abbreviations: CrPVd3, Cricket Paralysis Virus intergenic region internal ribosome entry site (IRES) domain 3 RNA; MPD, methylpentanediol; P4P6, the P4-P6 domain of the Tetrahymena group I intron; SRP, signal recognition particle.

Reprint requests to: Jeffrey S. Kieft, HHMI/Department of Biochemistry and Molecular Genetics, University of Colorado School of Medicine, Mail Stop 8101, PO Box 6511, Aurora, CO 80045, USA; e-mail: Jeffrey.Kieft@ucdenver.edu; fax: (303) 724-3215; or Barbara L. Golden, Department of Biochemistry, Purdue University, 175 South University Street, West Lafayette, IN 47907, USA; e-mail: barbgolden@purdue.edu; fax: (765) 494-7897.

Article published online ahead of print. Article and publication date are at http://www.rnajournal.org/cgi/doi/10.1261/rna.2072710. binding of anions has been reported in many crystal structures. However, it can be difficult to identify anions unambiguously within the constellation of solvent molecules and metal ions that surround the RNA (Das et al. 2001). This is largely because many of the anions used in crystallization experiments have electron densities that are similar to cations and solvent molecules present in the crystals. It has been proposed that anion binding sites in RNA crystal structures may correlate with locations ideal for interactions with negatively charged protein side chains or with the phosphates of nucleic acids (Masquida et al. 1999; Auffinger et al. 2004).

A study of anion binding sites in nucleic acid crystal structures was published in 2004 by Auffinger et al. (2004). In this study, 18 structures were used to analyze the binding sites of chloride, phosphate, and sulfate anions and to identify several potential binding motifs. These diverse sites were formed by the amine, N1, N3, and N7 of the purine bases, the amine and $\mathrm{N} 3$ of the $\mathrm{C}$ base, and the $2^{\prime}-\mathrm{OH}$ of the ribose sugar. In some cases these sites were reported as anion binding sites in the original studies, but in other cases they were reported as cations; Auffinger et al. (2004) suggest that the latter may be mislabeled or misidentified sites. Misidentification of anion binding sites could occur because binding of the negatively charged anions to the highly negatively charged RNA is counterintuitive and 
because the identity of these anions generally must be deduced from their chemical environment and electron density. In rare cases, when resolution is high enough $(<2.0 \AA)$, sulfates or phosphates can be identified from their tetrahedral geometry. As most structures of complex RNA molecules are only determined at modest resolution, many anion binding sites within crystal structures may be missed or assigned as cations (Das et al. 2001). Direct methods to identify bound anions have not been reported.

Here, we report the direct identification of anion binding sites within folded RNA structures using X-ray diffraction and the anomalous signal from selenate anions. Anions are found bound to RNA under conditions of both high ionic strength and more biologically relevant ionic conditions. The location of these bound selenates reveals that many of the previously proposed anion binding motifs are authentic. We do observe, however, that most of the anion binding motifs involve multiple stacked bases. These results confirm that localized anion binding sites exist in RNA and that selenate may be a useful general tool to identify many of them.

\section{RESULTS AND DISCUSSION}

\section{Bound selenate and sulfate anions identified by anomalous diffraction}

We recently solved the crystal structure of the Cricket Paralysis Virus intergenic region internal ribosome entry site domain 3 RNA (hereafter referred to as $\mathrm{CrPVd} 3$ ) by $\mathrm{X}$-ray crystallography (Costantino et al. 2008). Crystals of this RNA grow in high concentrations of $\mathrm{Li}_{2} \mathrm{SO}_{4}$, which interferes with binding of many heavy atoms that are useful to derivatize RNA crystals for phase determination. As part of the strategy to solve this structure, the crystals first were grown in high concentrations of $\mathrm{Li}_{2} \mathrm{SO}_{4}(1.4-1.5 \mathrm{M})$ and then transferred to a solution containing lithium acetate $\left(\mathrm{LiCH}_{3} \mathrm{CO}_{2}\right)$ and either cobalt (III) or iridium (III) hexammine. The manipulated crystals then were used to obtain phase information and refine the structure (PDB ID 3B31) (Keel et al. 2007; Costantino et al. 2008). The full rationale for this strategy is described elsewhere (Costantino et al. 2008). Subsequently, data collected from native, underivatized $\mathrm{CrPVd} 3$ crystals that were grown and cryoprotected in $\mathrm{Li}_{2} \mathrm{SO}_{4}$ were used to generate difference Fourier maps (Table 1). Examination of $1 \mathrm{~F}_{\mathrm{o}}-\mathrm{F}_{\mathrm{c}}$ difference Fourier maps from data sets obtained from two different sulfate-containing crystals (crystals CrPVd 3 \#1 and $\mathrm{CrPVd} 3$ \#2) reveals four strong areas of density located near the RNA that we labeled sites 1-4 (Fig. 1A). Because these areas of density are not present in density maps from $\mathrm{LiCH}_{3} \mathrm{CO}_{2}$-containing crystals but are visible in the maps generated from crystals cryoprotected with $\mathrm{Li}_{2} \mathrm{SO}_{4}$, we hypothesized that these areas of density were bound sulfate anions, but the modest resolution of the diffraction data precluded verification based on geometry.

To unambiguously identify these areas of density, we replaced $\mathrm{Li}_{2} \mathrm{SO}_{4}$ with $\mathrm{Li}_{2} \mathrm{SeO}_{4}$, either by directly growing the crystals in $\mathrm{Li}_{2} \mathrm{SeO}_{4}$ or by transferring crystals grown in $\mathrm{Li}_{2} \mathrm{SO}_{4}$ to $\mathrm{Li}_{2} \mathrm{SeO}_{4}$. Selenium is in the same chemical family as

TABLE 1. Crystallographic data collection statistics

\begin{tabular}{|c|c|c|c|c|c|}
\hline Crystal & P4-P6( $\Delta$ C209) & CrPVd3 \#1 & $\mathrm{CrPVd} 3 \# 2$ & $\mathrm{CrPVd} 3 \# 3$ & $\mathrm{CrPVd} 3 \# 4$ \\
\hline Anion & Selenate & Sulfate/native & Sulfate/native & Selenate & Selenate \\
\hline Resolution range $(\AA)$ & $20-2.9$ & $42-2.2$ & $49-1.9$ & $42-3.0$ & $50-3.1$ \\
\hline Wavelength $(\AA)$ & 0.9756 & 1.1271 & 1.1271 & 0.9786 & 0.9756 \\
\hline Space group & $\mathrm{P} 2{ }_{1}{ }_{1}{ }_{2}$ & $\mathrm{P} 4{ }_{1} 2_{1} 2$ & $\mathrm{P} 4{ }_{1} 2{ }_{1} 2$ & $\mathrm{P} 4{ }_{1} 2_{1} 2$ & $\mathrm{P} 4_{1} 2_{1} 2$ \\
\hline \multicolumn{6}{|l|}{ Unit cell } \\
\hline a $(\AA)$ & 75.12 & 58.94 & 59.28 & 59.21 & 59.63 \\
\hline $\mathrm{b}(\AA)$ & 125.4 & 58.94 & 59.29 & 59.21 & 59.63 \\
\hline $\mathrm{c}(\AA)$ & 145.2 & 99.06 & 99.72 & 99.70 & 99.35 \\
\hline$\alpha$ (degrees) & 90 & 90 & 90 & 90 & 90 \\
\hline$\beta$ (degrees) & 90 & 90 & 90 & 90 & 90 \\
\hline$\gamma$ (degrees) & 90 & 90 & 90 & 90 & 90 \\
\hline Total observations & 241,068 & 100,378 & 180,657 & 52,370 & 69,994 \\
\hline Unique reflections & 30,965 & 9451 & 14,084 & 6953 & 3580 \\
\hline Intensity/s intensity & 30.2 & 6.7 & 6.8 & 12.2 & 27.2 \\
\hline (last shell) & 2.6 & 2.3 & 2.0 & 4.7 & 7.2 \\
\hline Completeness (\%) & 99.4 & 100 & 99.9 & 99.9 & 99.4 \\
\hline (last shell) & 98.7 & 100 & 99.7 & 99.8 & 94.6 \\
\hline \multicolumn{6}{|l|}{ Rsym } \\
\hline overall & 6.4 & 22.2 & 22.3 & 9.6 & 15.0 \\
\hline (last shell) & 53.2 & 83.0 & 86.2 & 31.6 & 41.0 \\
\hline
\end{tabular}

$\mathrm{R}$ linear: SUM $[\mathrm{ABS}(\mathrm{I}-<\mathrm{I}>)]$ / SUM (I). 


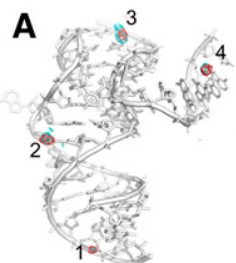

sulfate map
B

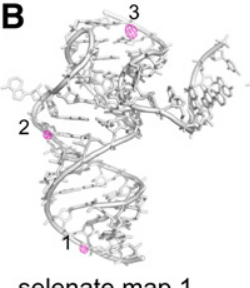

selenate map 1

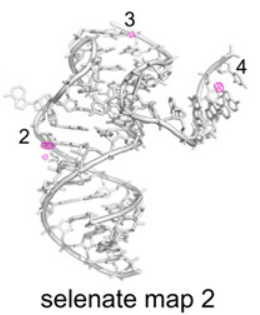

selenate map 2
FIGURE 1. Identification of bound $\mathrm{SO}_{4}{ }^{2-}$ and $\mathrm{SeO}_{4}{ }^{2-}$ anions. $(A)$ Two overlaid $1 \mathrm{~F}_{\mathrm{o}}-\mathrm{F}_{\mathrm{c}}$ difference maps generated using the structure of the CrPVd3 RNA (gray) solved using acetate-containing crystals and the diffraction data from two crystals containing $\mathrm{SO}_{4}{ }^{2-}$ (cyan from crystal CrPVd 3 \#1 and red from crystal CrPVd3 \#2). The four strong and distinct areas of density are labeled 1-4; they appear in both maps and, therefore, were preliminarily identified as $\mathrm{SO}_{4}{ }^{2-}$ anions. Maps are contoured at $4.5 \sigma$ and are rendered within $5 \AA$ of all RNA atoms. (B) Anomalous difference Fourier maps from two separate crystals containing $\mathrm{Li}_{2} \mathrm{SeO}_{4}{ }^{2-}$ (red and yellow) overlaid on the structure of the $\mathrm{CrPVd} 3$. Areas of density unambiguously indicate bound $\mathrm{SeO}_{4}{ }^{2-}$. Map 1 is from crystal CrPVd3 \#3 and map 2 is from crystal CrPVd3 \#4. Sites corresponding to the four putative $\mathrm{SO}_{4}{ }^{2-}$ sites in $A$ are labeled. Map 1 is contoured at $6 \sigma$ and map 2 at $5 \sigma$, and both are rendered within $5 \AA$ of all RNA atoms.

sulfur, positioned one row below it on the periodic table. Selenate and sulfate are isoelectronic, have similar bond lengths $(\sim 1.47 \AA$ for sulfate vs. $\sim 1.64 \AA$ for selenate) (Zalkin et al. 1964; Kolitsch 2002), and both anions interact with their ligands through tetrahedrally coordinated oxygen atoms. In these characteristics, sulfate and selanate are more similar than $\mathrm{Mg}^{2+}$ and $\mathrm{Mn}^{2+}$, which have different ligand preferences and therefore can have overlapping but also distinctly different binding sites. We hypothesized that the chemical and structural similarity of selenate and sulfate would allow them to bind to the same locations in the RNA. Diffraction data were collected at a wavelength corresponding to the $\mathrm{K}$ edge of selenium in its oxidized $\mathrm{SeO}_{4}{ }^{2-}$ state to maximize the anomalous diffraction signal (Table 1; Smith and Thompson 1998). These data were used with the refined structure (without modeled sulfate) to generate anomalous difference Fourier maps in which areas of density indicate selenium atoms. Maps from two separate data sets from two $\mathrm{SeO}_{4}{ }^{2-}$. containing crystals prepared using two different protocols were examined. These reveal unambiguous density in the locations of the four putative $\mathrm{SO}_{4}{ }^{2-}$ binding sites, although neither map contained all four sites (Fig. 1B). Specifically, $\mathrm{SeO}_{4}{ }^{2-}$ map 1 contains sites $1-3$ and $\mathrm{SeO}_{4}{ }^{2-}$ map 2 contains sites 2-4. The presence of anomalous difference density at these sites unambiguously confirms them as bound $\mathrm{SeO}_{4}{ }^{2-}$ anions, and by extension, the corresponding sites in the maps calculated with $\mathrm{SO}_{4}{ }^{2-}$-containing crystals are assigned to bound $\mathrm{SO}_{4}{ }^{2-}$.

\section{Binding of anions under low-salt conditions}

Crystals of CrPVd3 grow in buffers that are extremely high in ionic strength, 1.4-1.5 $\mathrm{M} \mathrm{Li}_{2} \mathrm{SO}_{4}$ (Costantino et al.
2008). These conditions are not physiological and anion binding to this RNA may be the result of these extreme buffer conditions. To investigate whether anion binding occurs in RNAs under low-ionic strength conditions, we explored anion binding to another RNA, the $\Delta$ C209 mutant of the P4-P6 domain of the Tetrahymena group I intron (P4-P6) (Juneau and Cech 1999; Juneau et al. 2001). This RNA crystallizes readily in physiologically relevant ionic strength conditions using methylpentanediol (MPD) as a precipitant. Crystals were soaked in a cryostabilization buffer containing $25 \mathrm{mM} \mathrm{Na}_{2} \mathrm{SeO}_{4}$ prior to freezing and data collection. Again, we used the ability to locate bound $\mathrm{SeO}_{4}{ }^{2-}$ anions (and by extension, $\mathrm{SO}_{4}{ }^{2-}$ binding sites) using anomalous diffraction. Anomalous difference Fourier maps contain several regions of density corresponding to bound selenate (Fig. 2A), suggesting that anions can bind to RNA under biologically relevant ionic strengths. These sites are present in both molecules in the asymmetric unit.

To summarize, the strategy of using $\mathrm{SeO}_{4}{ }^{2-}$ coupled to anomalous $\mathrm{X}$-ray diffraction analysis allowed us to assign the locations of bound $\mathrm{SeO}_{4}{ }^{2-}$ anions unambiguously in two different crystallized RNAs. The concentration of $\mathrm{SeO}_{4}{ }^{2-}$ present in these two crystals differed by more than an order of magnitude and similar binding sites were observed in both crystals. It is therefore unlikely that binding of these anions is an artifact of extremely high $\mathrm{SeO}_{4}{ }^{2-}$ concentrations. Rather, these sites likely represent authentic areas of
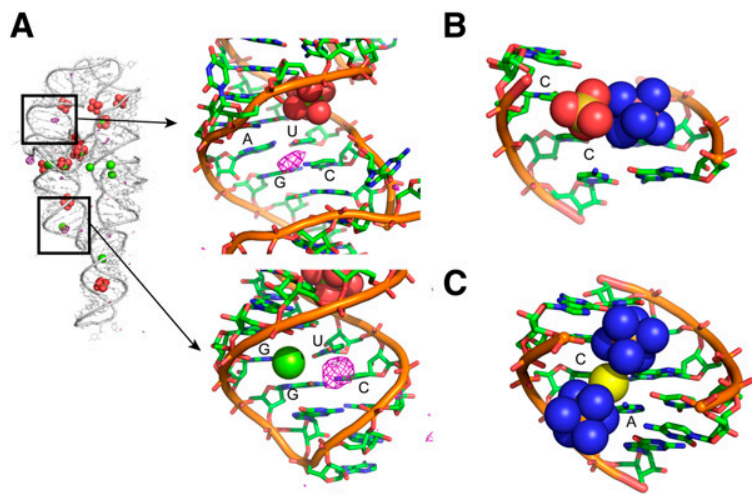

FIGURE 2. Coupled cation and anion binding in the major groove. (A) Crystal structure of the P4-P6 $\Delta 206$ domain of the Tetrahymena group I intron (PDB ID 1HR2) (Juneau et al. 2001) in the presence of $25 \mathrm{mM} \mathrm{SeO}_{4}{ }^{2-}$ and $25 \mathrm{mM} \mathrm{Mg}^{2+}$. Bound $\mathrm{Mg}^{2+}$ cations are shown in green with coordinated waters in red. An anomalous difference Fourier map contoured at $5 \sigma$ and rendered within $5 \AA$ of all atoms is overlaid in magenta. Two boxed regions show the locations of bound $\mathrm{SeO}_{4}{ }^{2-}$ in the vicinity of bound $\mathrm{Mg}^{2+}$ (sites 7 and 8), and the identities of neighboring bases are labeled. $(B)$ A portion of the hairpin ribozyme (PDB ID 2OUE) solved at $2.02 \AA$ resolution. A sulfate ion (red and yellow) is found bound in complex with a cobalt (III) hexammine ion (Salter et al. 2006). (C) A portion the structure of the E. coli SRP RNA bound to the M-domain protein (PDB ID 3LQX). Two cobalt (III) hexammine trivalent cations are shown in orange and blue, and an ion identified as $\mathrm{Cl}^{-}$is shown in yellow. The $\mathrm{Cl}^{-}$was identified on the basis of bond length, crystallographic temperature factor, and ligand identity. Bases that interact with the $\mathrm{Cl}^{-}$are labeled. 
local positive charge where the chemical environment is conducive to anion binding.

\section{A major groove anion binding motif}

In the $\mathrm{CrPVd} 3 \mathrm{RNA}, \mathrm{SeO}_{4}{ }^{2-}$ sites 1 and 3 are both within the major groove in the vicinity of tandem G-C pairs (both Gs on the same strand) and are positioned to interact with the two consecutive $\mathrm{C}$ bases (Fig. 3A). This binding position is similar to the CYT_CH site as defined by Auffinger et al. (2004); however, the authentic motif for these anion binding sites consists of more than one base or base pair rather than a single $\mathrm{C}$ as proposed by Auffinger et al. Major groove $\mathrm{SO}_{4}{ }^{2-}$ binding sites similar to sites 1 and 3 also were observed recently in the crystal structures of mutated sarcin/ricin loops from 23S rRNA (PDB IDs 1Q93, 1Q96) (Fig. 3B; Correll et al. 2003), the hairpin ribozyme (PDB ID 2OUE) (Fig. 2B; Salter et al. 2006), and the ligand-binding domain of the preQ(1) riboswitch (PDB ID 3GCA) (Fig. 3C; Spitale et al. 2009). These RNAs, like the CrPVd3 RNA, crystallized in high concentrations of $\mathrm{Li}_{2} \mathrm{SO}_{4}$ or $\left(\mathrm{NH}_{4}\right)_{2} \mathrm{SO}_{4}$. These sites involve two $\mathrm{C}$ bases of consecutive $\mathrm{G}-\mathrm{C}$ pairs as observed in the CrPVd3 RNA (Fig. 3A). A second class of site in which the sulfate binds in the major groove and interacts with the exocyclic amines on adjacent $\mathrm{C}-\mathrm{G}$ and $\mathrm{A}-\mathrm{U}$ base pairs are found in one structure of the sarcin/ricin loop (PDB ID 1Q93 but not 1Q96) (data not shown; Correll et al. 2003) and in the preQ(1) riboswitch (Fig. 3C).

The common feature among all of these sites is that they involve two bases and amine moieties in the major groove from these two adjacent bases, and this is consistent with

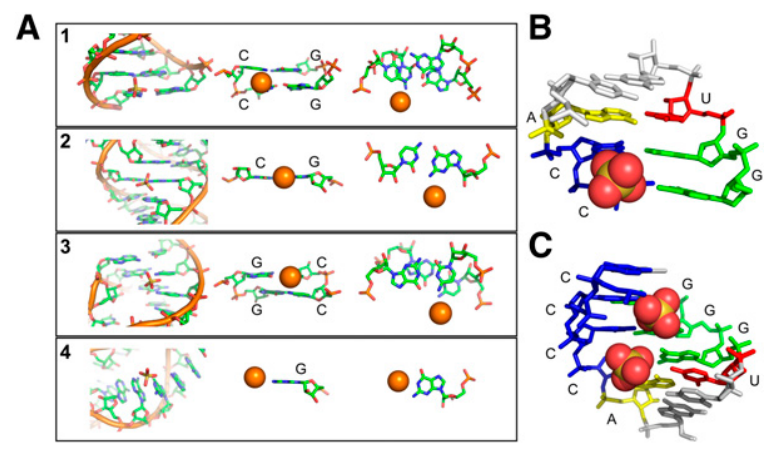

FIGURE 3. Details of the CrPVd3 RNA, sarcin/ricin loop, and preQ(1) riboswitch sulfate binding sites. $(A)$ The structural details of sulfate/ $\mathrm{SeO}_{4}{ }^{2-}$ binding sites 1-4 are shown. At left, the $\mathrm{SO}_{4}{ }^{2-}$ is shown in a stick model in the context of the CrPVd3 RNA structure. At middle and right, the $\mathrm{SO}_{4}{ }^{2-}$ anion is shown with just the nucleotides with which it interacts, viewed from two different orientations. The identity of interacting bases is labeled. For clarity, only the sulfur atom is shown as a sphere. Structures of $(B)$ sarcin/ricin loop from $23 S$ rRNA (PDB ID 1Q96) (Correll et al. 2003) and $(C)$ the ligand-binding domain of the preQ(1) riboswitch riboswitch (PDB ID 3GCA) (Spitale et al. 2009) with two $\mathrm{SO}_{4}{ }^{2-}$ binding sites shown. The sulfates are depicted as spheres in the major groove. Interacting bases are indicated, with $\mathrm{C}$ bases in blue, $\mathrm{G}$ bases in green, a $\mathrm{U}$ base in red, and an $\mathrm{A}$ base in yellow. the prediction that runs of consecutive $\mathrm{C}$ bases would be associated with anion binding sites (Auffinger et al. 2004). This is therefore likely a distinguishing feature of many RNA major groove anion binding sites and contrasts with major groove cation binding sites that often involve adjacent carbonyl groups of $\mathrm{G}$ and $\mathrm{U}$ bases (Cate et al. 1997; Kieft and Tinoco 1997; Masquida and Westhof 2000; Varani and McClain 2000; Keel et al. 2007).

\section{Anion binding sites outside the major groove}

Site 2 in the CrPVd3 is located in the minor groove where it interacts with the amine group of a $\mathrm{G}$ base (Fig. 3A). In this case the anion site appears to interact with a single base and is therefore similar to a GUA_S site previously described (Auffinger et al. 2004). Site 4 clearly involves only the Watson-Crick face of a single unpaired G base as no other base is positioned to interact with the anion (Fig. 3A). The anion can interact with both the amino and imino groups of the base; this type of anion binding motif is similar to the GUA_WC motif (Auffinger et al. 2004), and a similar site was observed in the mutant sarcin/ricin loop structure (data not shown; Correll et al. 2003).

\section{Anion binding sites often occur near cation binding sites}

In the P4-P6 $\Delta$ C209 RNA, two strong $\mathrm{SeO}_{4}{ }^{2-}$ binding sites are observed, both within the P5abc subdomain (Fig. 2A). There are two molecules in the asymmetric unit of these crystals, and a bound $\mathrm{SeO}_{4}{ }^{2-}$ ion is observed at the same sites in both molecules. These two sites share two features. First, in both sites the $\mathrm{SeO}_{4}{ }^{2-}$ is positioned to interact with the $\mathrm{C}$ in a G-C pair. Second, each of these anions is bound in close proximity to a bound magnesium cation in the major groove. These observations show that anion binding in RNA structures can be proximal to cation binding in the major groove. While we do not observe cations bound in the $\mathrm{CrPVd} 3$ structure, these crystals contained $>4 \mathrm{M}$ lithium. These lithium ions do not possess sufficient electron density to be visible, and they likely displaced bound divalent cations. However, in the P4-P6 structure, the presence of $25 \mathrm{mM} \mathrm{MgCl} 2$ and lower monovalent concentration allows the divalent cations to bind in discrete locations and to be observed crystallographically.

Additional evidence for cations and anions binding together in the RNA major groove is found in the aforementioned hairpin ribozyme structure, where the bound sulfate is accompanied by a bound trivalent cobalt (III) hexammine cation (PDB ID 2OUE) (Fig. 2B; Salter et al. 2006). Also, the structure of the E. coli SRP RNA bound to the M-domain protein (PDB ID 3LQX) contains a chloride ion bound between two cobalt (III) hexamine trivalent cations (Fig. 2B; Batey et al. 2000; Batey and Doudna 2002). The identity of the chloride was inferred by the presence of chloride in the 
crystallization solution, the low crystallographic B-factor (compared with water), and the chemical environment ( $\mathrm{R}$ Batey, pers. comm.). Specifically, six amine groups are positioned within $3.2-3.5 \AA$ from the putative chloride, including one from a $\mathrm{C}$ base and one from an $\mathrm{A}$. The position of this chloride is likely dictated by these base functional groups, and the strong positive charge of the two proximal cations: It is attracted to both cations and likely facilitates the close approach of these two cations. Similar "electrostatic sandwiches" are seen in some protein active sites (Jiang et al. 2002). Hence, it is likely that anion and cation binding are coupled in this site; our data suggests this may be a theme that applies to many other ion-RNA interactions in the major groove. If true, the combined effects of cations and anions may be important for stabilizing the fold of complex RNA structures.

\section{Selenate as a tool}

The ability to observe the anomalous signal from $\mathrm{SeO}_{4}{ }^{2-}$ anions bound to RNA is a potentially useful tool for studying RNA structure. By soaking $\mathrm{SeO}_{4}{ }^{2-}$ into RNA crystals and collecting anomalous diffraction data, anion binding sites potentially can be distinguished from water or cation binding sites, and putative anion binding sites can be identified. This can reduce ambiguity in assigning electron density in X-ray structures. In the case of the P4-P6 crystals, $\mathrm{SeO}_{4}{ }^{2-}$ binding was observed at only $25 \mathrm{mM} \mathrm{SeO}_{4}{ }^{2-}$ concentration and, hence, the high levels of $\mathrm{SO}_{4}{ }^{2-}$ or $\mathrm{SeO}_{4}{ }^{2-}$ present in the other structures (CrPVd3, preQ(1) riboswitch, sarcin/ricin loop) may not be necessary to induce binding of anions to authentic sites. In addition, we observed $\mathrm{SeO}_{4}{ }^{2-}$ binding at motifs previously reported to bind phosphate (GUA_WC), sulfate (GUA_WC, CYT_CH, GUA_S), and chloride (GUA_WC, CYT_CH, GUA_S) anions. Thus, selenate may be a useful general probe for sites capable of binding other anions (Auffinger et al. 2004), although binding sites specific for certain anion species may also exist.

Unlike selenium, phosphorus and chloride do not have $\mathrm{X}$-ray absorption edges at wavelengths that are readily observable by most synchrotron light sources; however, they are potentially detectable by several means. First, these anions are expected to have ligand geometries that differ significantly from those of water and magnesium ions. Second, these anions are expected to have a preponderance of nitrogenous ligands from the RNA. Third, when modeled as a water, these ions will often have an unrealistically low B-factor. Fourth, although the signal is significantly weaker than selenium, phosphorus and chlorine can be observed by anomalous scattering using a diffraction data set obtained at longer wavelengths (at $8000 \mathrm{eV}, \mathrm{f}^{\prime \prime}$ equals $0.71 \mathrm{e}$ and $0.44 \mathrm{e}$ for chlorine and phosphorus, respectively). Finally, bromide, like selenate, has an X-ray absorption edge that can be used to identify binding sites for halide ions using anomalous difference Fourier methods. Unfortunately, attempts to identify bromide binding sites in the P4-P6 $\Delta 206$ domain yielded ambiguous results (E Chase and BL Golden, unpubl.).

\section{Conclusions}

Anions bind to RNA. Although localized bound anions are often found in crystal structures in the presence of very high anion concentrations, we unambiguously observe $\mathrm{SeO}_{4}{ }^{2-}$ bound at lower concentrations as well. This suggests that anions can bind in solution at physiological concentrations. Binding sites are found at positively charged regions: exocyclic amines of $\mathrm{A}$ and $\mathrm{C}$ and metal ions. The biological relevance of these anions remains unclear, however. They may work in concert with cations to help stabilize RNA structure, they may indicate interaction sites for protein side chains or phosphates, and they could modulate intermolecular interactions (Auffinger et al. 2004). The use of $\mathrm{SeO}_{4}{ }^{2-}$ to identify anion binding sites provides a useful tool for future explorations of anion-nucleic acid interactions, anion-protein interactions, and may even be a tool to obtain phases to solve RNA crystal structures.

\section{MATERIALS AND METHODS}

\section{Crystallization and data collection}

The transcription, purification, and crystallization of CrPVd3 RNA is described in detail elsewhere (Keel et al. 2007; Costantino et al. 2008). To generate native, sulfate-containing crystals of this RNA, a solution of $5 \mathrm{mg} / \mathrm{mL}$ RNA, $10 \mathrm{mM} \mathrm{K-HEPES} \mathrm{(} \mathrm{pH} 7.5$ ), $2.5 \mathrm{mM}$ $\mathrm{MgCl}_{2}$, and $0.5 \mathrm{mM}$ spermidine- $\mathrm{HCl}$ was heated to $65^{\circ} \mathrm{C}$ for $3 \mathrm{~min}$ and allowed to cool on the bench. This was combined at a 1:1 ratio $(1 \mu \mathrm{L}+1 \mu \mathrm{L})$ with $1.4 \mathrm{M} \mathrm{Li}_{2} \mathrm{SO}_{4}, 40 \mathrm{mM} \mathrm{Mg}\left(\mathrm{CH}_{3} \mathrm{CO}_{2}\right)_{2}, 50 \mathrm{mM}$ HEPES-NaOH ( $\mathrm{pH}$ 7.5) in a hanging-drop/vapor diffusion experiment at $30^{\circ} \mathrm{C}$. Crystals appeared in a few days, and then grew over the course of several weeks to maximum dimensions. To cryoprotect, the well solution was replaced with $2.4 \mathrm{M} \mathrm{Li}_{2} \mathrm{SO}_{4}$ and the well was resealed and allowed to equilibrate for at least $24 \mathrm{~h}$. The crystals CrPVd3 \#1 and CrPVd3 \#2 were harvested from the drop and cooled directly in liquid nitrogen. Data were collected at beamline 8.2.1 at the Advanced Light Source (Table 1).

The $\mathrm{SeO}_{4}{ }^{2-}$-containing crystal $\mathrm{CrPVd} 3$ \#3 was grown as above except the $\mathrm{Li}_{2} \mathrm{SO}_{4}$ was replaced with $1.8 \mathrm{M} \mathrm{Li}_{2} \mathrm{SeO}_{4}$ and was cryoprotected by replacing the well solution with $2.4 \mathrm{M} \mathrm{Li}_{2} \mathrm{SO}_{4}$ as described above. CrPVd3 \#4 was grown in $1.4 \mathrm{M} \mathrm{Li}_{2} \mathrm{SO}_{4}$ and then transferred into saturated $\mathrm{Li}_{2} \mathrm{SeO}_{4}$. Crystals were harvested and flash-cooled in liquid nitrogen. Data for crystal CrPVd3 \#3 were collected at beamline 4.2.2 of the Advanced Light Source and data for crystal CrPVd3 \#4 were collected at beamline 22-ID at the Advanced Photon Source (Table 1).

Crystals of the P4-P6 $\Delta 206$ domain were produced essentially as previously described (Juneau et al. 2001). A 1-2 $\mu \mathrm{L}$ vol of a solution containing $4 \mathrm{mg} / \mathrm{mL}$ RNA, $10 \mathrm{mM} \mathrm{NaCl}, 25 \mathrm{mM} \mathrm{MgCl}$, and $5 \mathrm{mM}$ HEPES-KOH ( $\mathrm{pH} 7.5$ ) was mixed with a $1-2 \mu \mathrm{L}$ vol of precipitant containing $50 \mathrm{mM}$ K-cacodylate $\mathrm{pH} 6.5,0.5 \mathrm{mM}$ spermine, and 18\%-20\% MPD. The resulting drop was equilibrated against the precipitant in a hanging-drop/vapor diffusion 
experiment at $18^{\circ} \mathrm{C}$. Crystals were soaked in 25\% MPD, $20 \%$ isopropanol, $50 \mathrm{mM}$ potassium cacodylate $(\mathrm{pH}$ 6.5), $1 \mathrm{mM}$ spermine, $25 \mathrm{mM} \mathrm{MgCl}_{2}, 25 \mathrm{mM} \mathrm{Na}_{2} \mathrm{SeO}_{4}$ for $2 \mathrm{~h}$ prior to flashcooling in liquid nitrogen. Crystallographic data were collected at beamline 14-ID-B at the Advanced Photon Source (Table 1).

\section{Diffraction data processing, molecular replacement, and refinement}

Diffraction data were integrated and scaled using either $\mathrm{d}^{\star}$ trek (Pflugrath 1999) or HKL2000 (Otwinowski and Minor 1997). The structures of CrPVd3 \#1, CrPVd3 \#2, CrPVd3 \#3, and CrPVd3 \#4 were solved by molecular replacement using Phaser (McCoy et al. 2007) with a search model that consisted of PDB entry 3B31 with water and ions removed (Costantino et al. 2008). The solution was refined with CNS (Brunger et al. 1998) by energy minimization. Sulfates or selenates were added as their binding sites were identified, and additional refinement was conducted. The final $\mathrm{R} / \mathrm{R}_{\text {free }}$ of CrPVd3 \#2 was 24.2/27.6, of CrPVd3 \#3 was 23.2/28.4, and of CrPVd3 \#4 was 22.7/26.3 (CrPVd3 \#1 data was not used in refinement). Data from the P4-P6 crystals was integrated and scaled with HKL2000 (Otwinowski and Minor 1997). The selenium anomalous difference map was calculated with CNS (Brunger et al. 1998) using phases from the model 1HR2 (Juneau et al. 2001). Coordinates have been deposited in the Protein Data Bank with accession numbers $3 \mathrm{MJB}, 3 \mathrm{MJa}$, and $3 \mathrm{MJ} 3$.

\section{ACKNOWLEDGMENTS}

We thank the staffs of Advanced Light Source Beamlines 8.2.1 and 4.2.2 and BioCars for assistance with data collection; Aimee Keel, James Whitehurst, Phil Bevilacqua, and Rob Batey for critical reading of this manuscript; and Rob Batey, Joe Wedekind, Carl Correll, and Jeff Bolin for helpful discussions. This work was supported by NIH grant R01GM072560 to J.S.K., the Purdue Center for Cancer Research; and the Purdue Department of Biochemistry. J.S.K. is a Howard Hughes Medical Institute Early Career Scientist.

Received December 31, 2009; accepted February 17, 2010.

\section{REFERENCES}

Auffinger P, Westhof E. 1998. Hydration of RNA base pairs. J Biomol Struct Dyn 16: 693-707.

Auffinger P, Bielecki L, Westhof E. 2004. Anion binding to nucleic acids. Structure 12: 379-388.

Batey RT, Doudna JA. 2002. Structural and energetic analysis of metal ions essential to SRP signal recognition domain assembly. Biochemistry 41: 11703-11710.

Batey RT, Rambo RP, Lucast L, Rha B, Doudna JA. 2000. Crystal structure of the ribonucleoprotein core of the signal recognition particle. Science 287: 1232-1239.

Brunger AT, Adams PD, Clore GM, DeLano WL, Gros P, GrosseKunstleve RW, Jiang JS, Kuszewski J, Nilges M, Pannu NS, et al. 1998. Crystallography \& NMR system: A new software suite for macromolecular structure determination. Acta Crystallogr D Biol Crystallogr 54: 905-921.
Cate JH, Hanna RL, Doudna JA. 1997. A magnesium ion core at the heart of a ribozyme domain. Nat Struct Biol 4: 553-558.

Correll CC, Beneken J, Plantinga MJ, Lubbers M, Chan YL. 2003. The common and the distinctive features of the bulged-G motif based on a 1.04 A resolution RNA structure. Nucleic Acids Res 31: 68066818.

Costantino DA, Pfingsten JS, Rambo RP, Kieft JS. 2008. tRNA-mRNA mimicry drives translation initiation from a viral IRES. Nat Struct Mol Biol 15: 57-64.

Das U, Chen S, Fuxreiter M, Vaguine AA, Richelle J, Berman HM, Wodak SJ. 2001. Checking nucleic acid crystal structures. Acta Crystallogr D Biol Crystallogr 57: 813-828.

Draper DE. 2004. A guide to ions and RNA structure. RNA 10: 335343.

Jiang YL, Drohat AC, Ichikawa Y, Stivers JT. 2002. Probing the limits of electrostatic catalysis by uracil DNA glycosylase using transition state mimicry and mutagenesis. J Biol Chem 277: 1538515392.

Juneau K, Cech TR. 1999. In vitro selection of RNAs with increased tertiary structure stability. RNA 5: 1119-1129.

Juneau K, Podell E, Harrington DJ, Cech TR. 2001. Structural basis of the enhanced stability of a mutant ribozyme domain and a detailed view of RNA-solvent interactions. Structure 9: 221-231.

Keel AY, Rambo RP, Batey RT, Kieft JS. 2007. A general strategy to solve the phase problem in RNA crystallography. Structure 15: 761-772.

Kieft JS, Tinoco I Jr. 1997. Solution structure of a metal-binding site in the major groove of RNA complexed with cobalt (III) hexammine. Structure 5: 713-721.

Kolitsch U. 2002. Magnesium selenate hexahydrate, MgSeO4.6H2O. Acta Crys E 58: i3-i5.

Masquida B, Westhof E. 2000. On the wobble GoU and related pairs. RNA 6: 9-15.

Masquida B, Sauter C, Westhof E. 1999. A sulfate pocket formed by three GoU pairs in the 0.97 A resolution X-ray structure of a nonameric RNA. RNA 5: 1384-1395.

McCoy AJ, Grosse-Kunstleve RW, Adams PD, Winn MD, Storoni LC, Read RJ. 2007. Phaser crystallographic software. J Appl Crystallogr 40: $658-674$.

Otwinowski Z, Minor W. 1997. Processing of X-Ray diffraction data collected in oscillation mode. Methods Enzymol 276: 307-326.

Pflugrath JW. 1999. The finer things in X-ray diffraction data collection. Acta Crystallogr D Biol Crystallogr 55: 1718-1725.

Pyle AM. 2002. Metal ions in the structure and function of RNA. J Biol Inorg Chem 7: 679-690.

Salter J, Krucinska J, Alam S, Grum-Tokars V, Wedekind JE. 2006. Water in the active site of an all-RNA hairpin ribozyme and effects of Gua8 base variants on the geometry of phosphoryl transfer. Biochemistry 45: 686-700.

Smith JL, Thompson A. 1998. Reactivity of selenomethionine-dents in the magic bullet? Structure 6: 815-819.

Spitale RC, Torelli AT, Krucinska J, Bandarian V, Wedekind JE. 2009. The structural basis for recognition of the PreQ0 metabolite by an unusually small riboswitch aptamer domain. J Biol Chem 284: 11012-11016.

Sundaralingam M, Pan B. 2002. Hydrogen and hydration of DNA and RNA oligonucleotides. Biophys Chem 95: 273-282.

Varani G, McClain WH. 2000. The G x U wobble base pair. A fundamental building block of RNA structure crucial to RNA function in diverse biological systems. EMBO Rep 1: 18-23.

Woodson SA. 2005. Metal ions and RNA folding: A highly charged topic with a dynamic future. Curr Opin Chem Biol 9: 104-109.

Zalkin A, Ruben H, Templeton DH. 1964. The crystal structure and hydrogen bonding of magnesium sulfate hexahydrate. Acta Crys 17: $235-240$. 

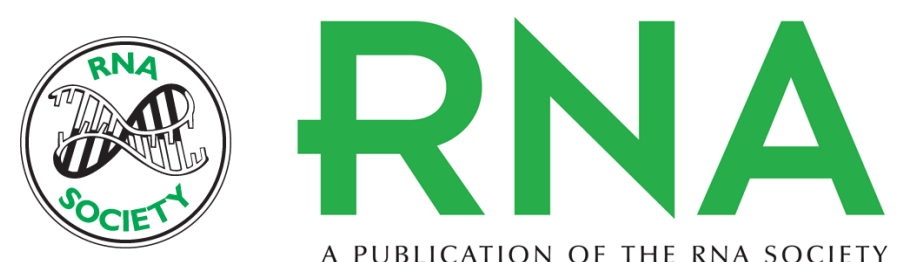

A PUBLICATION OF THE RNA SOCIETY

\title{
Identification and characterization of anion binding sites in RNA
}

\author{
Jeffrey S. Kieft, Elaine Chase, David A. Costantino, et al.
}

RNA 2010 16: 1118-1123 originally published online April 21, 2010

Access the most recent version at doi:10.1261/rna.2072710

\section{References This article cites 29 articles, 7 of which can be accessed free at: http://rnajournal.cshlp.org/content/16/6/1118.full.html\#ref-list-1}

\section{License}

Email Alerting Receive free email alerts when new articles cite this article - sign up in the box at the Service top right corner of the article or click here. 